\title{
On the dynamics of the collapse of a diffusion-flame hole
}

\author{
By C. PANTANO AND D. I. PULLIN \\ Graduate Aeronautical Laboratories, 105-50, California Institute of Technology, \\ Pasadena, CA, 91125, USA \\ cpantano@galcit.caltech.edu
}

(Received 19 September 2002 and in revised form 14 November 2002)

The collapse dynamics of a diffusion-flame hole in the presence of a counterflow are studied. We construct unsteady solutions of the one-dimensional edge-flame model of Buckmaster (1996), in which heat and mass transverse losses are algebraic. The flame structure is determined in the classical limit of large activation energy. Solutions for both planar and axisymmetric strain geometry are considered for the particular case of unity Lewis number. It is shown that the final stage of the edge-flame collapse is determined by a dominant balance between the time rate of change of the mass fractions (and temperature) and diffusion, giving a self-similar structure in which the size of the edge-flame hole approaches zero, to leading (zeroth) order, as a $\frac{1}{2}$-power of time. This solution suggests an expansion of the full model equations in $\frac{1}{2}$-powers of time that allows detailed analysis of the effects of side losses and flow distribution in the edge-flame collapse process. It is found that side loss effects are apparent at the first order, whereas convection by the counterflow is first felt during collapse at the second order in the fractional-time expansion. Numerical integrations of the governing equations are found to verify the analytic results.

\section{Introduction}

The process leading to the formation of diffusion-flame holes is fairly well understood. When the rate of strain is sufficiently large, diffusion of heat from the reaction zone exceeds the rate of heat released by chemical conversion. At the same time, one of the reactants typically leaks through the flame until the flame is locally quenched. A hole in the flame surface then appears. The region where the transition takes place from a fully burning state to a quenched state is commonly referred to as the flame edge. The structure of the flame edge depends, among other things, on the Damköhler number, a flow to chemical characteristic time scale ratio. When this is large, a socalled triple-flame structure forms, composed of two premixed flames attached to the nose of a following diffusion flame in a form that resembles an arrow. At lower Damköhler numbers, a simple edge-flame can occur. When a hole is formed, it is of interest to know the conditions governing hole growth or shrinkage and collapse. The importance of flame hole dynamics has been identified by Williams (1985).

Detailed numerical studies have been carried out in freely propagating edge-flames with and without the associated effects of heat release by Kioni et al. (1993), Ruetsch, Vervisch \& Liñán (1995) and Echekki \& Chen (1996). Theoretical descriptions of triple edge-flames using the large activation energy asymptotic approximation with finite heat release were developed by Ghosal \& Vervisch (2000). In these cases, 
there is a well-defined velocity, referred to here as the free edge-flame velocity, that depends on the Damköhler and Lewis numbers and the level of heat release. Some studies have considered the interaction of the edge-flame with a counterflow that is perpendicular to the plane of the flame, also called a strained mixing layer (Daou \& Liñán 1998; Vedarajan \& Buckmaster 1998; Buckmaster \& Short 1999; Thatcher \& Dold 2000; Short, Buckmaster \& Kochevets 2001). Shay \& Ronney (1998) studied the effects of variable strain rate in space. The experimental configuration corresponds to opposed jets that are slightly misaligned in order to generate the desired strain field. They show the formation of stable edge-flames and observed that the value of the strain rate at the edge of the flame was almost always lower than the value of the extinction strain rate for a uniform non-premixed flame with the same reactants, and this was independent of the stain-rate gradient. Moreover, their measurements compared favourably with theoretical predictions from a model due to Buckmaster (1996). In the case of triple-edge flames, Ko \& Chung (1999) performed experiments with methane-air jets and reported that their unsteady edge flames propagate at a speed that increases with decreasing mixture fraction gradient, in agreement with theoretical predictions. This velocity is higher than the stoichiometric laminar burning velocity. Their measurements show that there is a high correlation between the edgeflame speed and the flame curvature. Santoro, Liñán \& Gomez (2000) have performed experimental measurements of methane-air flames in a counterflow mixing layer and find the existence of standing edge-flames, with triple-flames for large Damköhler number and simple edge-flames for lower Damköhler numbers. This occurs in the region where the flow is decelerated away from the stagnation region and leads to the formation of stable structures.

Buckmaster \& Jackson (2000) investigated the effects of curvature and time evolution of collapsing and expanding flame holes and disks. In their study, the two-dimensional time-dependent transport equations governing a single-step reaction under a constant density approximation, were solved numerically. For the case of a collapsing flame hole, these solutions show that the trajectories of edge velocity plotted against the instantaneous distance between the flame edge and the centre of the hole, all collapse towards an enveloping curve after a short initial transient time that depends on the initial conditions. Similar results were obtained for both planar and axisymmetric hole geometry, although the envelope curve appeared to have different numerical values in each case. As discussed in Buckmaster \& Jackson (2000), the curvature term that appears in the axisymmetric case has an indirect effect on the numerical values of the enveloping state. The numerical solutions suggest that the envelope state is the result of the interaction of the opposed edges of the flame, that in the numerical context appears as the symmetry condition imposed at the hole centre. It is of interest to understand the mechanism responsible for this generic state of hole collapse, and this is the purpose of the present paper.

From a theoretical point of view, the one-dimensional model of Buckmaster (1996) is attractive because it approximates the equations governing the dynamics of flame edges by a set of one-dimensional transport equations. In this model, heat and species losses to the side are modelled as algebraic functions. Furthermore, Nayagam \& Williams (2002) have used a version of this model in which the inner structure of the edge-flame is analogous to the premixed flame of Liñán (1974). Depending on the values of the parameters, a freely propagating edgeflame can propagate with or against the local flow direction, or can even remain stationary (Buckmaster 1996; Nayagam \& Williams 2002). The dynamics of flame holes (Nayagam, Balasubramaniam \& Ronney 1999) and flame disks (Nayagam \& 
Williams 2001) have been considered in previous studies by neglecting the effect of the flow using one-dimensional models. Nayagam et al. (1999) analysed the dynamics of axisymmetric flame holes, without a background flow interacting with the flame edge. They showed that these free flame-holes are unstable for any $D a$. Only when the flame-hole is anchored by a heat sink is it stable, and only then provided that the initial hole radius is sufficiently small. They further show that for sufficiently large $D a$, the free flame-hole always heals itself; it collapses, driven by the heat generated at the flame edge which is always larger than the losses through the boundaries.

In the present paper, we address the dynamics of opposed edge-flames in a counterflow by studying the unsteady solutions of the one-dimensional model of Buckmaster. Both planar and axisymmetric geometries are considered in which one principal strain-rate axis of the counter flow is perpendicular to the plane of the flame. As will be seen, our interest is primarily in the dynamics of small flame holes. Here, we refer to hole diameters that are small with respect to the thickness of the mixing layer, not to be confused with the flame thickness which is much smaller in our case of large activation energies. This is motivated by the fact that these holes may be generated in turbulent flames when the rate of strain (or scalar dissipation rate), a random variable, exceeds the quenching value at one point of the flame surface for a sufficient duration of time such that a hole is formed. Subsequently, if the rate of strain diminishes below the quenching value and the hole shrinks and collapses, we would like to know the physical mechanisms that control the dynamics of the collapse. We address questions concerning the temporal structure of the final stages of flame hole collapse. To make the analysis tractable we consider the particular case of unit Lewis number of both the fuel and the oxidizer. Both analytical and numerical solutions of the one-dimensional mode equations are considered.

\section{Formulation}

The flow and flame geometry is shown in figure 1 . The uniform two-dimensional linear straining flow has velocity components $(a r,-(1+j) a z)$, and since $a>0$, the flow diverges to infinity on the $r$-axis. The parameter $j$ takes the values $j=0$ for the planar configuration and $j=1$ for the axisymmetric case. We now consider the thin-flame limit, in which the flame thickness is much smaller than any length scale in the radial direction. The problem can now be transformed into a one-dimensional problem by integrating the two-dimensional governing equations over the vertical span of the flame thickness. The one-dimensional flame model, due to Buckmaster (1996), always has the flame lying on $z=0$, in $\left|r_{f}(t)\right| \leqslant|r|<\infty$ with symmetry about $r=0$. This is a simplifying assumption introduced to make the problem tractable analytically; in a more physically realistic model, the flame edge has a two-dimensional edge structure. Far from the hole, the one-dimensional diffusion flame will conform to a planar structure and this makes the simplified structure of the edge desirable. In this work, we assume that the Damköhler number is not too large so that only simple edge flames result at the edges of the hole. The oxidizer stream extends from $z=-\infty$ to 0 and the fuel stream extends from $z=0$ to $\infty$. We will assume that the thermochemical variables are functions only of $r$ and time $t$, and lateral losses are modelled algebraically (Buckmaster 1996). The equations describing the fuel mass fraction and temperature in $0 \leqslant r<\infty, t>0$ are then

$$
\rho \frac{\partial T}{\partial t}+\rho a r \frac{\partial T}{\partial r}-\frac{\lambda}{c_{p}}\left(\frac{\partial^{2} T}{\partial r^{2}}+\frac{j}{r} \frac{\partial T}{\partial r}\right)=\frac{Q}{c_{p}} C_{1} \dot{\Omega}-\frac{C_{1} \lambda}{c_{p} l^{2}}\left(T-T_{w}\right),
$$




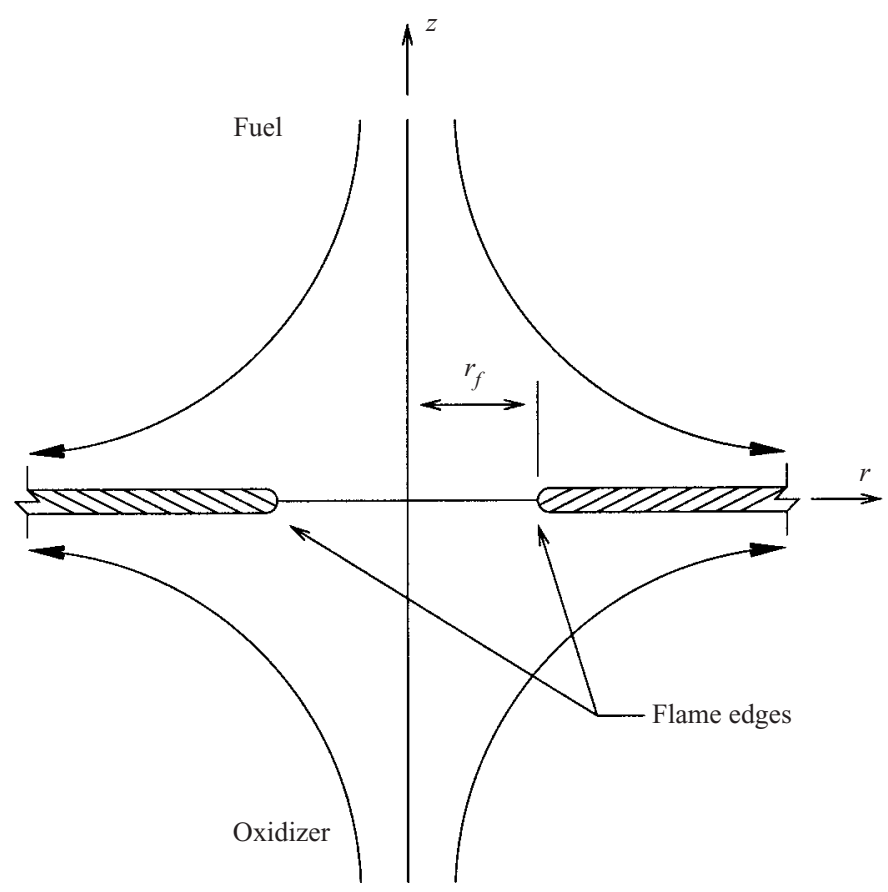

Figure 1. Edge-flame geometry.

and the oxidizer and fuel mass fractions are governed by

$$
\begin{gathered}
\rho \frac{\partial X}{\partial t}+\rho a r \frac{\partial X}{\partial r}-\rho D_{X}\left(\frac{\partial^{2} X}{\partial r^{2}}+\frac{j}{r} \frac{\partial X}{\partial r}\right)=-\gamma_{X} C_{2} \dot{\Omega}+\frac{C_{2} \rho D_{X}}{l^{2}}\left(X_{w}-X\right), \\
\rho \frac{\partial Y}{\partial t}+\rho a r \frac{\partial Y}{\partial r}-\rho D_{Y}\left(\frac{\partial^{2} Y}{\partial r^{2}}+\frac{j}{r} \frac{\partial Y}{\partial r}\right)=-\gamma_{Y} C_{3} \dot{\Omega}+\frac{C_{3} \rho D_{Y}}{l^{2}}\left(Y_{w}-Y\right),
\end{gathered}
$$

respectively, where $X$ is the oxidizer mass fraction, $Y$ is the fuel mass fraction, $T$ is the temperature, $l$ is the thickness of the mixing layer and $\rho$ is the density. The parameters $T_{W}, X_{W}$ and $Y_{W}$ denote the free-stream values of $T, X$ and $Y$, respectively. These values are assumed constants to be specified. Both free stream, fuel and oxidizer, are assumed to enter the domain at the same temperature, $T_{W}$. The parameters $\gamma_{X}$ and $\gamma_{Y}$ are the stoichiometric coefficients of oxidizer and fuel, respectively. The constants $C_{i}$ should be assigned to obtain the correct stoichiometric conditions and must be considered here as parameters of the side loss model. Additionally, $\rho, \rho D_{i}, \lambda / c_{p}$ and $c_{p}$ are assumed constant, where $D_{Y}$ and $D_{X}$ are the diffusivity of the fuel and oxidizer, respectively, $\lambda$ is the thermal conductivity of the mixture, and $c_{p}$ is the specific heat at constant pressure of the mixture. The heat release parameter $Q / c_{p}$ is assumed constant and the reaction rate is modelled by

$$
\dot{\Omega}=\rho^{2} K\left(X-X_{a}\right)\left(Y-Y_{a}\right) \mathrm{e}^{-E / R T},
$$

where $E$ is the activation energy of the reaction and $K$ is the characteristic rate prefactor. As described in Buckmaster (1996), the Burke-Schumann temperature of the system is defined as $T_{a}$. This is a constant of the problem that depends on the stoichiometry and thermodynamic constants of the system. The terms $X_{a}$ and $Y_{a}$ are the linear equilibrium mass fractions, obtained for the Burke-Schumann case of 
infinite Damköhler number. These terms are piecewise linear functions of $T_{a}$ and represent average oxidizer and fuel mass fractions at the diffusion flame sheet in the present model. In this context, we must understand (2.4) as a closure model of the reaction rate term for the averaged temperature, oxidizer and fuel mass fractions across the flame. The boundary conditions are

$$
\begin{gathered}
\frac{\partial X}{\partial r}=\frac{\partial Y}{\partial r}=\frac{\partial T}{\partial r}=0, \quad r=0, \\
Y \rightarrow Y_{a}, \quad X \rightarrow X_{a}, \quad T \rightarrow T_{a}, \quad r \rightarrow \infty,
\end{gathered}
$$

for infinite Damköhler number. The system of equations, (2.1)-(2.4), can be written in non-dimensional form using

$$
\theta=\frac{T}{T_{a}}, \quad \tilde{X}=\frac{X}{\gamma_{X}}, \quad \tilde{Y}=\frac{Y}{\gamma_{Y}}, \quad s=\frac{r}{l}, \quad \tau=t a,
$$

where we assume that the diffusivities of all species are equal, $D_{X}=D_{Y}=D$, and $L e=\lambda / \rho D c_{p}$ is the Lewis number which we take equal to one for simplicity. In this flow, the thickness of the mixing layer established between the two opposed streams is proportional to $\sqrt{\lambda / \rho c_{p} a}$ (Santoro et al. 2000) and without loss of generality we take the proportionality constant equal to one, that is

$$
l=\sqrt{\frac{\lambda}{\rho c_{p} a} .}
$$

The dimensionless equations are now

$$
\begin{gathered}
\frac{\partial \theta}{\partial \tau}+s \frac{\partial \theta}{\partial s}-\left(\frac{\partial^{2} \theta}{\partial s^{2}}+\frac{j}{s} \frac{\partial \theta}{\partial s}\right)=C_{1}\left(\theta_{W}-\theta\right)+C_{1} q \dot{\omega}, \\
\frac{\partial \tilde{X}}{\partial \tau}+s \frac{\partial \tilde{X}}{\partial s}-\left(\frac{\partial^{2} \tilde{X}}{\partial s^{2}}+\frac{j}{s} \frac{\partial \tilde{X}}{\partial s}\right)=C_{2}\left(\tilde{X}_{W}-\tilde{X}\right)-C_{2} \dot{\omega},
\end{gathered}
$$

and

$$
\frac{\partial \tilde{Y}}{\partial \tau}+s \frac{\partial \tilde{Y}}{\partial s}-\left(\frac{\partial^{2} \tilde{Y}}{\partial s^{2}}+\frac{j}{s} \frac{\partial \tilde{Y}}{\partial s}\right)=C_{3}\left(\tilde{Y}_{W}-\tilde{Y}\right)-C_{3} \dot{\omega},
$$

with

$$
\dot{\omega}=\Delta\left(\tilde{X}-\tilde{X}_{a}\right)\left(\tilde{Y}-\tilde{Y}_{a}\right) \mathrm{e}^{(1-1 / \theta) / \epsilon} .
$$

The heat release parameter is defined as

$$
q=\frac{Q}{c_{p} T_{a}} .
$$

The small quantity, $\epsilon$, is defined as

$$
\epsilon=\frac{R T_{a}}{E}
$$

and the Damköhler number is

$$
\Delta=\frac{\rho K \gamma_{X} \gamma_{Y}}{a} \mathrm{e}^{-1 / \epsilon}
$$


The boundary conditions in dimensionless form are

$$
\begin{gathered}
\frac{\partial \tilde{X}}{\partial s}=\frac{\partial \tilde{Y}}{\partial s}=\frac{\partial \theta}{\partial s}=0, \quad s=0, \\
\tilde{X} \rightarrow \tilde{X}_{a}, \quad \tilde{Y} \rightarrow \tilde{Y}_{a}, \quad \theta \rightarrow 1, \quad s \rightarrow \infty,
\end{gathered}
$$

in the limit of infinite Damköhler number, while the initial conditions are

$$
\tilde{X}=\tilde{X}_{o}(s), \quad \tilde{Y}=\tilde{Y}_{o}(s), \quad \theta=\theta_{o}(s), \quad \tau=0 .
$$

We wish to obtain $\tilde{X}(s, \tau), \tilde{Y}(s, \tau), \theta(s, \tau)$ and $s_{f}(\tau)$. Since $s_{f}(\tau)$ must be determined, there is implicit nonlinearity reminiscent of the Steffan problem in heat transfer. In the limit of large activation energy asymptotics, the flame structure comprises an inner solution where finite rate chemistry is important, sandwiched by two outer zones. In the quenched zone, the reaction rate term is negligible. In the steadily burning zone, the reaction rate term is in stoichiometric balance with the transverse supply of fuel and oxidizer.

\subsection{Outer problem}

We denote the outer solution in $0 \leqslant s<s_{f}$ the 'left' solution, and that in $s_{f}<s<\infty$ as the 'right' solution. In $0 \leqslant s<s_{f}$ (left), the extinguished region, $\dot{\omega}$ can be neglected since the reaction is frozen because the temperature is low compared to $T_{a}$. In $s_{f}<s<\infty$ (right), changes in $\tilde{X}, \tilde{Y}$ and $\theta$ in distances of order $s$ are negligible, and so the right-hand sides of (2.5)-(2.7) must be equal to zero. This condition gives

$$
\frac{\theta-\theta_{W}}{q}=\tilde{X}_{W}-\tilde{X}=\tilde{Y}_{W}-\tilde{Y} .
$$

For large Damköhler numbers, we also have, by construction, that $\tilde{X} \rightarrow \tilde{X}_{a}, \tilde{Y} \rightarrow \tilde{Y}_{a}$ and $\theta \rightarrow 1$ and (2.12) yields

$$
\frac{1-\theta_{W}}{q}=\tilde{X}_{W}-\tilde{X}_{a}=\tilde{Y}_{W}-\tilde{Y}_{a},
$$

that in combination with (2.8) gives a single equation for $\theta$ as a function of $\Delta$ and $\epsilon$. This equation,

$$
\theta-\theta_{W}=\frac{\Delta}{q}(1-\theta)^{2} \mathrm{e}^{(1-1 / \theta) / \epsilon},
$$

describes the S-shape dependence of temperature on Damköhler number. The upper branch of this curve corresponds to the near-equilibrium regime described by Liñan (1974) in which $\theta \approx 1$. For small values of $\epsilon$, the near-equilibrium branch can be obtained by expanding the non-dimensional temperature as

$$
\theta=1-\epsilon^{n} \theta_{n},
$$

where $n$ is unknown at this point, but it is positive. Introducing (2.15) into (2.14) and collecting terms gives

$$
1-\theta_{W}-\epsilon^{n} \theta_{n}=\frac{\Delta}{q} \epsilon^{2 n} \theta_{n}^{2} \exp \left(-\epsilon^{(n-1)} \theta_{n}\right) .
$$

By analysing the structure of the thin reaction zone at the flame edge, we find that the case of interest is that for which the Damköhler number is proportional to $\epsilon^{-3}$ 
(Buckmaster 1996). We define then an order one Damköhler number, $\Delta_{1}$, such that

$$
\Delta=\frac{\Delta_{1}}{\epsilon^{3}} \text {. }
$$

Introducing (2.17) into (2.16) and neglecting the last term on the left-hand side (because, as will be seen, $n>1$ ), expanding the exponential and retaining the leadingorder term gives

$$
\theta_{n}=\sqrt{\frac{q\left(1-\theta_{W}\right)}{\Delta_{1}}}
$$

with $n=\frac{3}{2}$. Using (2.12)-(2.13), the top branch equilibrium solutions are given by

$$
\theta=1-\epsilon^{3 / 2} \theta_{n}, \quad \tilde{Y}=\tilde{Y}_{a}+\epsilon^{3 / 2} \frac{\theta_{n}}{q}, \quad \tilde{X}=\tilde{X}_{a}+\epsilon^{3 / 2} \frac{\theta_{n}}{q} .
$$

\subsection{Inner problem}

Within the large-activation-energy formalism, the burning in the flame edge is always stronger, of order $\epsilon^{2}$, than that in the diffusion flame, of order $\epsilon^{3}$. This can be shown by first writing $\tilde{X}, \tilde{Y}$ and $\theta$ in terms of the stretched coordinate

$$
\xi=\left(s-s_{f}\right) / \epsilon,
$$

and expanding the dependent variables as

$$
\begin{gathered}
\theta=1+\epsilon \theta_{1}+O\left(\epsilon^{3 / 2}\right), \\
\tilde{X}=\tilde{X}_{a}+\epsilon \tilde{X}_{1}+O\left(\epsilon^{3 / 2}\right), \\
\tilde{Y}=\tilde{Y}_{a}+\epsilon \tilde{Y}_{1}+O\left(\epsilon^{3 / 2}\right) .
\end{gathered}
$$

Substituting these expressions into (2.5)-(2.7) and retaining the leading-order terms in $\epsilon$ gives

$$
\begin{gathered}
\frac{\mathrm{d}^{2} \theta_{1}}{\mathrm{~d} \xi^{2}}=-C_{1} q \Delta_{1} \tilde{X}_{1} \tilde{Y}_{1} \exp \left(\theta_{1}\right), \\
\frac{\mathrm{d}^{2} \tilde{X}_{1}}{\mathrm{~d} \xi^{2}}=C_{2} \Delta_{1} \tilde{X}_{1} \tilde{Y}_{1} \exp \left(\theta_{1}\right), \\
\frac{\mathrm{d}^{2} \tilde{Y}_{1}}{\mathrm{~d} \xi^{2}}=C_{3} \Delta_{1} \tilde{X}_{1} \tilde{Y}_{1} \exp \left(\theta_{1}\right) .
\end{gathered}
$$

All other terms, those representing the time rate of change, convective transport, side losses and curvature, are negligible with respect to the second-order derivative term. In the case where the time derivative term is neglected, this is possible if the time rates of change of the temperature and mass fractions are not very large. More precisely, the condition $\epsilon^{2} \partial \theta_{1} / \partial \tau \ll 1$ must be satisfied. In the case of an edgeflame collapse, as will be shown, there will always be an instant in the collapse in which this condition will be violated. This is not a severe limitation since, within the large activation energy, asymptotic formalism $\epsilon$ can be made arbitrarily small. In the case of real flames, where $\epsilon$ is not too small, this limitation may play a role. A second limitation appears when the edge-flame radius is very small, for, in that case, the curvature term that was previously neglected, proportional to $1 / s$, cannot be discarded easily. This situation will appear when $s_{f}$ becomes of order $\epsilon$ and in 
the present analysis we will restrict the discussion to those cases for which $s_{f} \gg \epsilon$ because, among other things, the simple one-dimensional model of this study is then not appropriate; all thicknesses, flame hole and flame edge being similar, the problem becomes two-dimensional. A third limitation appears when the flame-edge velocity is very large. Indeed, the term that results from the convective change of variables (attached to the flame edge), of order $\dot{s}_{f} \epsilon$, must be small. This condition is ultimately violated when the velocity becomes very large; but again, since $\epsilon$ is arbitrarily small, the edge velocity restriction is not dominant until the very final stage of collapse.

Coupling the variables in the traditional Shvab-Zel'dovich form, that is, adding (2.23) divided by $q C_{1}$ to (2.24) divided by $C_{2}$, then gives a linear relationship between $\theta_{1}$ and $\tilde{X}_{1}$ whose constant of integration is determined by taking into account that $\tilde{X}_{1}, \tilde{Y}_{1}$ and $\theta_{1}$ go to zero as $\xi \rightarrow \infty$. The same integration is carried out for $\tilde{Y}_{1}$. The coupling relationships are then

$$
\tilde{X}_{1}=-C_{2} \theta_{1} /\left(C_{1} q\right), \quad \tilde{Y}_{1}=-C_{3} \theta_{1} /\left(C_{1} q\right) .
$$

These relationships can be now used in (2.23) to write a single differential equation that involves only $\theta_{1}$, namely

$$
\frac{\mathrm{d}^{2} \theta_{1}}{\mathrm{~d} \xi^{2}}=-D \theta_{1}^{2} \mathrm{e}^{\theta_{1}}
$$

where

$$
D=\frac{\Delta_{1} C_{2} C_{3}}{q C_{1}} .
$$

As shown by Buckmaster (1996), this equation can be integrated by introducing the change of variables $g\left(\theta_{1}\right)=\mathrm{d} \theta_{1} / \mathrm{d} \xi$ and using the facts that $\theta_{1}$ must tend to zero as $\xi \rightarrow \infty$ and that it takes large negative values at $\xi \rightarrow-\infty$. This provides a relationship between the temperature gradient at $\xi=-\infty$ and the chemistry parameters,

$$
\begin{gathered}
\left.\frac{\mathrm{d} \theta_{1}}{\mathrm{~d} \xi}\right|_{-\infty}=2 \sqrt{D}, \\
\left.\frac{\mathrm{d} \tilde{X}_{1}}{\mathrm{~d} \xi}\right|_{-\infty}=-\frac{C_{2}}{C_{1} q} 2 \sqrt{D}, \\
\left.\frac{\mathrm{d} \tilde{Y}_{1}}{\mathrm{~d} \xi}\right|_{-\infty}=-\frac{C_{3}}{C_{1} q} 2 \sqrt{D} .
\end{gathered}
$$

\section{The dynamics of diffusion-flame hole collapse}

In the previous section, we defined the complete problem and boundary conditions that govern this idealized non-stationary problem. Nayagam et al. (1999) have solved the equations for the axisymmetric case when the time rate of change of all species mass fraction and temperature is small by approximating the first term on the lefthand side of the transport equations by the convective change of variables,

$$
\frac{\partial}{\partial \tau} \rightarrow-\dot{s}_{f} \frac{\partial}{\partial s},
$$

and neglecting all convective losses. This approximation is warranted in the case where the time rate of change of the edge-flame position is small. We address now a more general case that can arise when the evolution of the edge-flame position is not necessarily slow. There is, nevertheless, a limitation imposed by the thin-flame 
regime considered in this study. In order to obtain a self-consistent one-dimensional problem, the convective-diffusive preheat length scale, of order $D / \dot{r}_{f}$, must be much larger than the flame thickness, of order $\epsilon l$. This implies that $\epsilon l \ll D / \dot{r}_{f}$, and in non-dimensional form this gives $\epsilon \dot{s}_{f} \ll 1$. Since $\epsilon$ is small, $\dot{s}_{f}$ need not be small all the time. Note that this approximation fails in the final instant of the collapse, when the edge velocity becomes very large (as will be shown), but, by this time, the opposed edges are so close that they interact and merge into a continuous diffusion flame.

We now consider the time-dependent transport equations, (2.5)-(2.7), in the region $0 \leqslant s \leqslant s_{f}$ with the reaction rate terms that appear on the right-hand side equal to zero and unity Lewis number. This is the problem that must be solved, to leading order, in the quenched region. In this case, it is well known that only one equation need be solved, the other variables being related through a linear coupling function. This equation can be cast in normalized form as

$$
\frac{\partial \varphi}{\partial \tau}+s \frac{\partial \varphi}{\partial s}-\left(\frac{\partial^{2} \varphi}{\partial s^{2}}+\frac{j}{s} \frac{\partial \varphi}{\partial s}\right)-C(1-\varphi)=0 \quad\left(s \leqslant s_{f}(\tau)\right),
$$

where we have used the symbol $\varphi$, defined as

$$
\varphi=\frac{1-\theta}{1-\theta_{W}} \quad \text { or } \quad \varphi=\frac{\tilde{X}-\tilde{X}_{a}}{\tilde{X}_{W}-\tilde{X}_{a}} \quad \text { or } \quad \varphi=\frac{\tilde{Y}-\tilde{Y}_{a}}{\tilde{Y}_{W}-\tilde{Y}_{a}},
$$

depending on the field of interest. From this point on, $\varphi$ will denote any of the normalized fields. The constant $C$ will take the value $C_{1}$ for the temperature, $C_{2}$ for the oxidizer mass fraction and $C_{3}$ for the fuel mass fraction, and as shown by Buckmaster (1996) they must take values determined from the stoichiometric conditions at the flame. In the present study of the dynamics of the flame hole collapse, it will be seen that a solution can be obtained for an arbitrary value of $C$. The boundary conditions for $\varphi$ are the condition of symmetry

$$
\frac{\partial \varphi}{\partial s}=0, \quad s=0
$$

the value of $\varphi$ at $s_{f}$, namely

$$
\varphi=0, \quad s=s_{f}(\tau),
$$

and the matching condition, (2.27)-(2.29), expressed in terms of $\varphi$

$$
\frac{\partial \varphi}{\partial s}=-\delta, \quad s=s_{f}(\tau)
$$

where

$$
\delta=\frac{2 \sqrt{D}}{\left(1-\theta_{W}\right)} \quad \text { or } \quad \delta=\frac{C_{2}}{C_{1} q} \frac{2 \sqrt{D}}{\left(\tilde{X}_{W}-\tilde{X}_{a}\right)} \quad \text { or } \quad \delta=\frac{C_{3}}{C_{1} q} \frac{2 \sqrt{D}}{\left(\tilde{Y}_{W}-\tilde{Y}_{a}\right)},
$$

is a reduced Damköhler number depending on the field being considered. From this point, all references to the Damköhler number refer to $\delta$. These boundary conditions must be complemented by an initial condition of the form

$$
\varphi(s, 0)=\varphi_{o}(s) .
$$

We are primarily interested in the temporal structure of the collapse of opposed edge-flames. Certainly, we have $s_{f} \rightarrow 0$ as $\tau \rightarrow \tau_{o}$, where $\tau_{o}$ is the time of collapse. We begin by supposing that the edge-flame collapse has a self-similar structure. In this case, we expect that, owing to the boundary conditions, $\varphi$ will approach zero as 
the edge flame collapses. This suggests that $\varphi$ can be expressed in terms of powers of $\left(\tau_{o}-\tau\right)$, that is

$$
\varphi=\left(\tau_{o}-\tau\right)^{v} y(\eta)
$$

with normalized edge-flame position given by

$$
s_{f_{0}}=c\left(\tau_{o}-\tau\right)^{\gamma},
$$

and where

$$
\eta=\frac{s}{s_{f_{0}}(\tau)}
$$

is a self-similar variable, $c$ is a constant and $v$ and $\gamma$ are exponents to be determined. The self-similar variable, $\eta$, has a clear physical representation, with a value of unity at the edge-flame position. Introducing these expressions into the boundary condition, (3.6), leads to

$$
\left(\tau_{o}-\tau\right)^{\nu-\gamma} \frac{\mathrm{d} y}{\mathrm{~d} \eta}(1)=-\delta .
$$

Since $\delta$ is a constant, we must have

$$
\gamma=v .
$$

Introducing (3.9) into (3.2) and using (3.11) and (3.13), gives

$$
-v\left(y-\eta \frac{\mathrm{d} y}{\mathrm{~d} \eta}\right)\left(\tau_{o}-\tau\right)^{(\nu-1)}=\left(\tau_{o}-\tau\right)^{-v}\left(\frac{\mathrm{d}^{2} y}{\mathrm{~d} \eta^{2}}+\frac{j}{\eta} \frac{\mathrm{d} y}{\mathrm{~d} \eta}\right)+C+\left(\tau_{o}-\tau\right)^{v}\left(-\frac{\mathrm{d} y}{\mathrm{~d} \eta}-C y\right) .
$$

The only non-trivial balance in this equation is obtained with the choice $v=\frac{1}{2}$. This suggests a general expansion in powers of $\left(\tau_{o}-\tau\right)^{1 / 2}$. The leading-order terms in (3.14), proportional to $\left(\tau_{o}-\tau\right)^{-1 / 2}$, correspond to a balance between the temporal rate of change of $\varphi$ and the diffusion terms. The next term, independent of time, contains the effect of the algebraic side losses. The following term in the expansion, proportional to $\left(\tau_{o}-\tau\right)^{1 / 2}$, contains the effect of the flow distribution and more side losses. We now formally obtain the leading-order collapse in self-similar variables. This is followed by a detailed expansion that addresses the effects of side losses and flow distribution.

\subsection{Leading-order solution}

We seek solutions of (3.2) for small hole sizes, $s_{f}(\tau) \ll 1$, that are of self-similar form, determined, to leading order, by dominant balance between the temporal term and the diffusion terms in (3.2). We therefore seek a solution of the form

$$
\varphi(s, \tau)=\delta s_{f_{0}}(\tau) y_{1}(\eta),
$$

where $\eta=s / s_{f_{0}}(\tau)$ is a self-similar variable and $s_{f_{0}}$ is the leading-order edge-flame position with time, given by

$$
s_{f_{0}}(\tau)=\sqrt{2 \Lambda\left(\tau_{o}-\tau\right)},
$$

where $\Lambda=-\dot{s}_{f_{0}} s_{f_{0}}$ is a constant to be determined, and $\dot{s}_{f}$ denotes the time derivative of $s_{f}$. Note that $\Lambda>0$, since physically relevant solutions must have $\dot{s}_{f}<0$. We remark that the presence of $\delta$ in (3.15) is adopted for convenience only (no assumption regarding the value of $\delta$ is made). Introducing (3.15) and (3.16) into the equation that 
results from neglecting side losses and flow effects in (3.2) gives

$$
-\Lambda\left(y_{1}-\eta y_{1}^{\prime}\right)=y_{1}^{\prime \prime}+\frac{j}{\eta} y_{1}^{\prime},
$$

with boundary conditions

$$
y_{1}^{\prime}(0)=0, \quad y_{1}(1)=0, \quad y_{1}^{\prime}(1)=-1,
$$

where the derivatives with respect to $\eta$ are denoted by primes. The parameter $\Lambda$ is an eigenvalue of the problem, determined by imposing the second boundary condition in (3.18).

The solutions to (3.17) belong, in general, to the family of confluent hypergeometric functions (Abramowitz \& Stegun 1972). This can be verified by considering the equation (of which (3.17) is a subset)

$$
F^{\prime \prime}+\frac{j}{\eta} F^{\prime}+\Lambda\left(\mathrm{d} F-\eta F^{\prime}\right)=0,
$$

and replacing $\zeta=\frac{1}{2} \Lambda \eta^{2}$. This equation is then transformed into Kummer's equation,

$$
\zeta \frac{\mathrm{d}^{2} F}{\mathrm{~d} \zeta^{2}}+(b-\zeta) \frac{\mathrm{d} F}{\mathrm{~d} \zeta}+\frac{1}{2} \mathrm{~d} F=0
$$

where

$$
b=\frac{1}{2}(1+j),
$$

and $d$ is a parameter. Equation (3.20) has general solutions of the form

$$
F(\eta)=c_{m} M\left(-\frac{1}{2} d, b, \frac{1}{2} \Lambda \eta^{2}\right)+c_{u} U\left(-\frac{1}{2} d, b, \frac{1}{2} \Lambda \eta^{2}\right),
$$

where $M$ and $U$ are the confluent hypergeometric functions. The solution for $y_{1}$ is obtained by setting $d=1$ in (3.21). Moreover, the solution that satisfies the symmetry boundary condition at $s=0$ is given by

$$
y_{1}(\eta)=c_{1} M\left(-\frac{1}{2}, b, \frac{1}{2} \Lambda \eta^{2}\right) .
$$

Requiring that $y_{1}(1)=0$, that is

$$
M\left(-\frac{1}{2}, b, \frac{1}{2} \Lambda\right)=0
$$

gives the value of the eigenvalue $\Lambda$. Finally, the value of $c_{1}$ is determined from the matching condition, $y_{1}^{\prime}(1)=-1$, and using a property of the derivative of a confluent hypergeometric function,

$$
\frac{\mathrm{d} M}{\mathrm{~d} z}(a, b, z)=\frac{a}{b} M(a+1, b+1, z),
$$

gives

$$
c_{1}=\frac{2 b}{\Lambda M\left(\frac{1}{2}, b+1, \frac{1}{2} \Lambda\right)} .
$$

The numeric values of $\Lambda$ and $c_{1}$ are given in table 1 .

\subsection{Effects of side losses and flow distribution}

It has been shown that, to leading or zeroth order, the final stages of the (self-similar) collapse are determined by the balance that exists between the time rate of change of $\varphi$ and diffusion. We address now the effects of side losses and the flow distribution. After careful consideration of the boundary conditions, we observe that it is convenient to 


\begin{tabular}{lcccccr}
\hline \multicolumn{1}{c}{ Case } & $\Lambda$ & $c_{1}$ & $c_{2}$ & $a_{2}$ & $A$ & \multicolumn{1}{c}{$B$} \\
Planar $(j=0)$ & 1.70807 & 0.425693 & 0.226581 & -0.453164 & 0.248365 & 0.175382 \\
Axisymmetric $(j=1)$ & 3.15991 & 0.390511 & 0.179193 & -0.262156 & 0.206543 & 0.074211 \\
TABLE 1. Numerical values of parameters determined from asymptotic expansion. The values \\
of $c_{2}, a_{2}, A$ and $B$ correspond to the case $C=1$.
\end{tabular}

perform an asymptotic expansion of the general solution in powers of $s_{f_{0}}(\tau)$. It can be verified that this is consistent and there are no intermediate powers of $\left(\tau_{o}-\tau\right)$ left unmatched in the expansion, as shown in (3.14). We seek then a solution of the form,

$$
\varphi=\delta s_{f_{0}}(\tau) y_{1}(\eta)+s_{f_{0}}^{2}(\tau) y_{2}(\eta)+\delta s_{f_{0}}^{3}(\tau) y_{3}(\eta)+O\left(s_{f_{0}}^{4}\right),
$$

with $\eta$ and $s_{f_{0}}(\tau)$ given by (3.11) and (3.16), respectively. Introducing (3.26) into (3.2) and collecting powers of $s_{f_{0}}$ gives,

$$
\begin{aligned}
-\Lambda\left(y_{1}-\eta y_{1}^{\prime}\right) & =y_{1}^{\prime \prime}+\frac{j}{\eta} y_{1}^{\prime}, \\
-\Lambda\left(2 y_{2}-\eta y_{2}^{\prime}\right) & =y_{2}^{\prime \prime}+\frac{j}{\eta} y_{2}^{\prime}+C, \\
-\Lambda\left(3 y_{3}-\eta y_{3}^{\prime}\right) & =y_{3}^{\prime \prime}+\frac{j}{\eta} y_{3}^{\prime}-\eta y_{1}^{\prime}-C y_{1} .
\end{aligned}
$$

Equation (3.27) was the objective of the preceding subsection and is the leadingorder solution. Equation (3.28), first-order correction, shows the effect of side losses. Equation (3.29) shows that the flow has second-order effects on the dynamics of the flame hole collapse.

To close (3.27)-(3.29), we must derive the respective boundary conditions. To this end, the flame-edge evolution is assumed to be of the form

$$
s_{f}=s_{f_{0}}+\frac{a_{2}}{\delta} s_{f_{0}}^{2}+\frac{a_{3}}{\delta^{2}} s_{f_{0}}^{3}+O\left(s_{f_{0}}^{4}\right) .
$$

Again, the appearance of $\delta$ in this expression is only for convenience since $\delta$ is not assumed to be either large or small. The boundary condition $\varphi\left(s_{f}, \tau\right)=0$ is expressed as a Taylor series expansion around $s_{f_{0}}$. Introducing (3.26) and (3.30) and gathering terms of the same order, gives

$$
\begin{gathered}
y_{1}(1)=0, \\
y_{1}^{\prime}(1) a_{2}+y_{2}(1)=0, \\
y_{1}^{\prime}(1) a_{3}+y_{1}^{\prime \prime}(1) \frac{1}{2} a_{2}^{2}+y_{2}^{\prime}(1) a_{2}+\delta^{2} y_{3}(1)=0 .
\end{gathered}
$$

The same expansion applied to the boundary condition $\partial \varphi / \partial s=-\delta$ at $s=s_{f}$ gives

$$
\begin{gathered}
y_{1}^{\prime}(1)=-1, \\
y_{1}^{\prime \prime}(1) a_{2}+y_{2}^{\prime}(1)=0, \\
y_{1}^{\prime \prime}(1) a_{3}+y_{1}^{\prime \prime \prime}(1) \frac{1}{2} a_{2}^{2}+y_{2}^{\prime \prime}(1) a_{2}+\delta^{2} y_{3}^{\prime}(1)=0 .
\end{gathered}
$$

The solution $y_{2}(\eta)$ is obtained from (3.28) with $y_{2}^{\prime}(0)=0$ and gives

$$
y_{2}(\eta)=c_{2} M\left(-1, b, \frac{1}{2} \Lambda \eta^{2}\right)-\frac{C}{2 \Lambda} .
$$


The boundary condition at $\eta=1$ can be written as

$$
y_{1}^{\prime \prime}(1) y_{2}(1)+y_{2}^{\prime}(1)=0
$$

obtained by eliminating $a_{2}$ from (3.32) and (3.35), and gives the value of $c_{2}$. Once this value is known, $a_{2}=y_{2}(1)$ is readily available. Table 1 gives the values of these constants.

The solution of $y_{3}(\eta)$ is obtained from (3.29) with $y_{3}^{\prime}(0)=0$ and gives

$$
y_{3}(\eta)=c_{3} y_{3}^{m}(\Lambda, \eta)+y_{p}(\eta) .
$$

The particular solution is obtained by the technique of variation of parameters, and gives

$$
y_{p}(\eta)=y_{3}^{u}(\Lambda, \eta) \int_{0}^{\eta} q_{1}\left(\eta^{*}\right) \frac{y_{3}^{m}\left(\Lambda, \eta^{*}\right)}{W\left(\Lambda, \eta^{*}\right)} \mathrm{d} \eta^{*}-y_{3}^{m}(\Lambda, \eta) \int_{0}^{\eta} q_{1}\left(\eta^{*}\right) \frac{y_{3}^{u}\left(\Lambda, \eta^{*}\right)}{W\left(\Lambda, \eta^{*}\right)} \mathrm{d} \eta^{*}
$$

where

$$
q_{1}(\eta)=c_{1}\left(C M\left(-\frac{1}{2}, b, \frac{1}{2} \Lambda \eta^{2}\right)-\frac{\Lambda \eta^{2}}{2 b} M\left(\frac{1}{2}, b+1, \frac{1}{2} \Lambda \eta^{2}\right)\right),
$$

and $y_{3}^{m}$ and $y_{3}^{u}$ are the two independent solutions of the homogeneous differential equation, (3.29), given by

$$
\begin{gathered}
y_{3}^{m}(\Lambda, \eta)=M\left(-\frac{3}{2}, b, \frac{1}{2} \Lambda \eta^{2}\right), \\
y_{3}^{u}(\Lambda, \eta)=U\left(-\frac{3}{2}, b, \frac{1}{2} \Lambda \eta^{2}\right) .
\end{gathered}
$$

In (3.39), $W$ denotes the Wronskian, given by

$$
W(\Lambda, \eta)=y_{3}^{m}(\Lambda, \eta) y_{3}^{\prime u}(\Lambda, \eta)-y_{3}^{\prime m}(\Lambda, \eta) y_{3}^{u}(\Lambda, \eta) .
$$

The boundary condition at $\eta=1$ can be written as

$$
y_{1}^{\prime \prime}(1) y_{3}(1)+y_{3}^{\prime}(1)=\frac{A}{\delta^{2}},
$$

where

$$
A=-\frac{1}{2} a_{2}\left(y_{1}^{\prime \prime}(1)\left(a_{2} y_{1}^{\prime \prime}(1)+2 y_{2}^{\prime}(1)\right)+a_{2} y_{1}^{\prime \prime \prime}(1)+2 y_{2}^{\prime \prime}(1)\right),
$$

and

$$
a_{3}=y_{1}^{\prime \prime}(1) \frac{1}{2} a_{2}^{2}+y_{2}^{\prime}(1) a_{2}+\delta^{2} y_{3}(1)=B+\delta^{2} y_{3}(1) \text {. }
$$

The constant $c_{3}$ is determined from (3.44). Once $y_{3}$ is known, $a_{3}$ is calculated from (3.46). Notice that it is not possible to obtain $c_{3}$ and $a_{3}$ independently of $\delta$. The reduced Damköhler number appears in an intricate way in the matching and cannot be factored out algebraically. The only values that are independent of $\delta$ are $A$ and $B$ (see table 1).

\subsection{Numerical solution}

The solution of the free-boundary problem corresponding to (3.2) and boundary and initial conditions, (3.4)-(3.8), determine $\varphi(s, \tau)$ and $s_{f}(\tau)$. These functions have been obtained numerically using a finite-difference technique. Spatial derivatives are discretized by second order of accuracy finite differences. The time derivative is approximated by a first order of accuracy Euler step. The treatment of the boundary condition at $s=0$ is straightforward. The boundary condition at $s=s_{f}(\tau)$ deserves further discussion. Differentiating the boundary condition (3.5) with respect to time 
and using (3.6), gives

$$
\frac{\partial}{\partial \tau} \varphi\left(s_{f}(\tau), \tau\right)=\frac{\partial \varphi}{\partial s} \dot{s}_{f}+\frac{\partial \varphi}{\partial \tau}=-\delta \dot{s}_{f}+\frac{\partial \varphi}{\partial \tau}=0 .
$$

Introducing (3.2), evaluated at $s=s_{f}$, to express the partial derivative of $\varphi$ with respect to time into (3.47), gives

$$
\dot{s}_{f}=\frac{C}{\delta}+s_{f}-\frac{j}{s_{f}}+\frac{1}{\delta} \frac{\partial^{2} \varphi}{\partial s^{2}} .
$$

Notice that (3.48) is not closed because the last term on the right-hand side of (3.48) (that is evaluated at $s=s_{f}$ ) can only be determined once the solution to the free-boundary problem is known.

The formulation of the numerical scheme uses (3.48) with the second-order partial derivative evaluated at the current time step to compute $\dot{s}_{f}$ and advance the edgeflame position to the next time step. In order to keep the same relative accuracy throughout the calculation, the number of points used in the spatial discretization is maintained fixed and equal to $N$ with a grid spacing defined as $\Delta s$. After each time step, the new $\varphi$ profile is interpolated onto a grid with constant spacing and the same number of points, $N$, but where the last point of the grid coincides with the new value of $s_{f}$. This is achieved by a polynomial interpolation of second order, i.e. the same order as the spatial discretization. The variable time step, $\Delta \tau$, is required to satisfy the stability limit of the discretized (3.2) and such that $\left|\dot{s}_{f} \Delta \tau\right|<\frac{1}{2} \Delta s$, in order to avoid large errors in the integration of $s_{f}$ associated with the moving boundary. In all calculations, the number of points was kept constant and equal to $N=500$, which proved to give accurate results, including during the edge-flame collapse.

The choice of the initial condition is arbitrary because, in principle, any function with the appropriate limits could be used. Here, we adopt the following strategy: it is shown in the Appendix that the present problem admits stationary solutions, although unstable, of the form

$$
\varphi(C, s)=1-\frac{M\left(\frac{1}{2} C, b, \frac{1}{2} s^{2}\right)}{M\left(\frac{1}{2} C, b, \frac{1}{2} s_{f}^{2}\right)},
$$

for values of $s_{f}$ that satisfy the matching condition, (3.6), that in the unity Lewis number case reduces to

$$
\delta^{*}=-\frac{\mathrm{d} \varphi}{\mathrm{d} s}\left(s_{f}\right) .
$$

For each value of $C$ and $s_{f}$, there is a unique value of the Damköhler number, that we denote here as $\delta^{*}$, for which stationary solutions exist in theory. As discussed by Santoro et al. (2000), this solution does not materialize in practice because these structures are unstable. If $\delta$ is not equal to the value given by (3.50), $\varphi$ of (3.49) is not a stationary solution and will evolve in time, together with $s_{f}$, according to the free-boundary problem described above. In the present study, we decided to choose the initial condition equal to (3.49) with $s_{f}$ replaced by $s_{f}(0)$, the initial edge-flame position, giving

$$
\varphi_{o}(C, s)=1-\frac{M\left(\frac{1}{2} C, b, \frac{1}{2} s^{2}\right)}{M\left(\frac{1}{2} C, b, \frac{1}{2} s_{f}^{2}(0)\right)} .
$$

Furthermore, unless stated otherwise, the value of $C$ appearing in (3.51) is the same as that appearing in (3.2) and was taken equal to one, the results are not sensitive to the value of $C$, provided it is a quantity of order one. The numerical integration of 


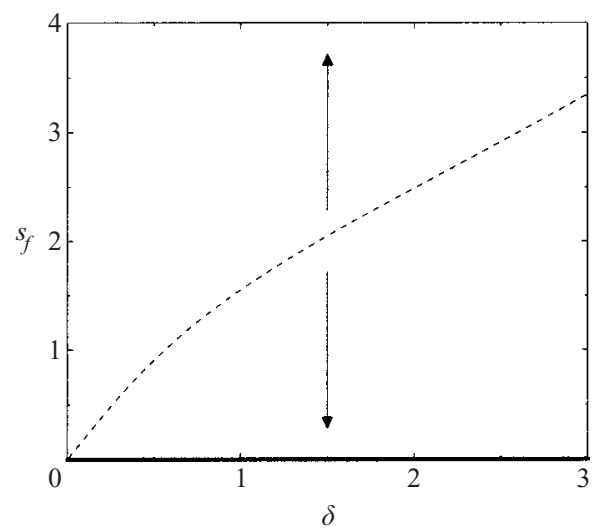

FIGURE 2. Evolution diagram for axisymmetric edge-flames in counterflow. The thick line represents the collapsed hole and the discontinuous line represents the unstable state $(C=1)$.

the free-boundary problem was carried out with the initial condition given by (3.51) for values of $\delta$ that differ from $\delta^{*}\left(C, s_{f}(0)\right)$.

As shown by Buckmaster \& Jackson (2000), the results for the axisymmetric case are topologically identical to those for the planar case. For that reason, we will present and discuss only the results corresponding to the axisymmetric case, keeping in mind that the same conclusions apply for the planar case with numerical values that are different. No additional physics were revealed by the planar case. From a more practical point of view, the axisymmetric case is more interesting because of its relevance to real diffusion-flame holes.

Figure 2 shows a topological diagram that depicts the evolution of the edge-flame position, $s_{f}(\tau)$, with time obtained from the numerical integration of the free-boundary problem for the axisymmetric case. The broken line represents the stationary solution, (3.50). The horizontal axis is represented as a thick line because it represents a collapsed edge flame, but it is not part of the stationary edge-flame solutions. There is no edge flame in this case, just a continuous diffusion flame. The arrows indicate the evolution of the edge-flame position with time in the different regions. Each point in these diagrams represents a complete free-boundary problem. Figure 2 shows that there is always only one possible stationary solution, independent of the value of $\delta$, but integration of (3.2) showed that, as expected, this stationary solution is not observed. The stationary branch is a repulsive state and represents a bifurcation between collapsing and expanding edge flames. For initial values of $s_{f}$ below the stationary solution, the edge flame will collapse, and vice versa, for initial $s_{f}$ larger than the stationary solution, the edge flame will expand. In previous studies, which did not take into account the effect of the flow (Nayagam et al. 1999; Nayagam \& Williams 2001), expanding and collapsing states were predicted on the basis of static stability arguments or analytical approximations of the free-boundary problem for slow-moving flame edges. Those results seem to apply here.

In the cases where the edge flame collapses, there is an associated time to collapse, $\tau_{o}$, denoting the time from the initial instant, $\tau=0$, up to the time of collapse. This characteristic time, that is obtained from the numerical solution and varies from case to case, is addressed next. Figure 3 shows the evolution of $s_{f}$ with time and various Damköhler numbers. All cases start with the same initial edge-flame position, $s_{f}(0)=2$, and the Damköhler number varies from a low value of 0.2 up to a 


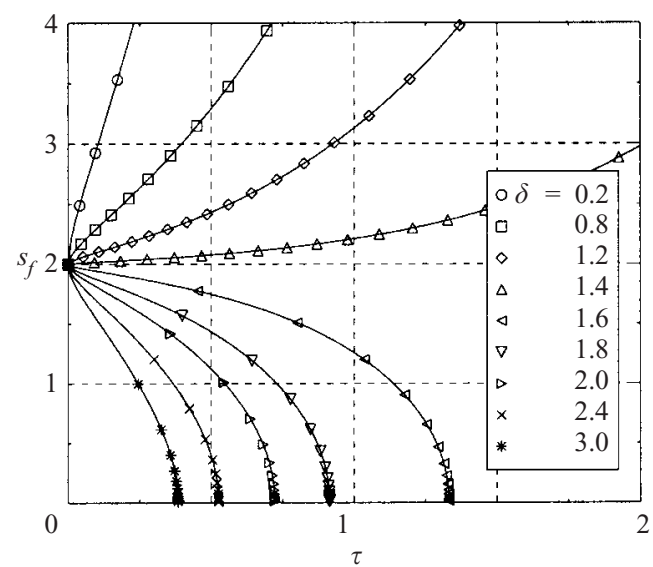

FIGURE 3. Evolution of $s_{f}$ with time for the axisymmetric case with $s_{f}(0)=2, C=1$.
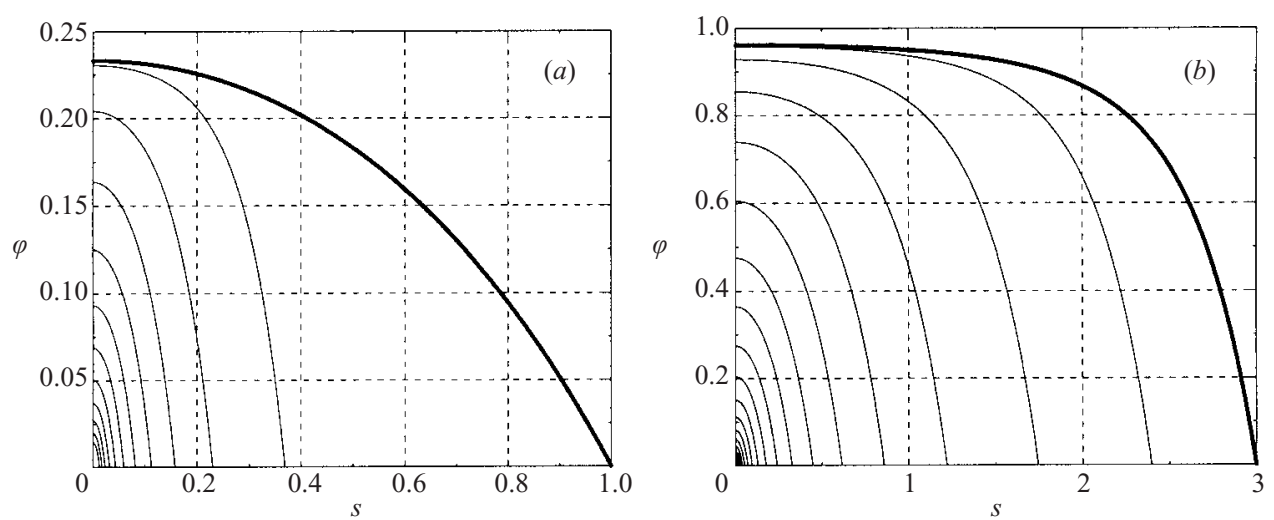

FIGURE 4. Evolution with time of $\varphi$ for axisymmetric case of two collapsing edge flames with $C=1$ and $\delta=3,(a) s_{f}(0)=1$ and $(b) s_{f}(0)=3$.

maximum value of 3 . The expanding and collapsing edge-flame solutions are clearly visible here. Comparing the profiles in figure 3 , it can be seen that the dynamics of $s_{f}$ are related to the distance between the initial condition and the stationary solution in figure 2. In other words, the larger the initial difference between $\delta$ and $\delta^{*}$, the faster is the dynamic evolution. If $\delta$ is close to $\delta^{*}$, the value of $\tau_{o}$ tends to be large. On the other hand, if $\delta$ is very different from $\delta^{*}$, the time to collapse, $\tau_{o}$, tends to be small. Since the difference between $\delta$ and $\delta^{*}$ measures the departure from the fixed point, the observed changes of $\tau_{o}$ are not entirely surprising. A rapid evolution is expected if the initial conditions are far from the fixed point and vice versa.

We turn our attention to the evolution of $\varphi$ profiles with time. Figure 4 shows the evolution with time of $\varphi$ profiles for two cases with $\delta=3$ and with initial edge-flame positions $s_{f}(0)=1$ and 3 . The thick curves correspond to the initial profiles and the subsequent profiles correspond to consecutive increasing times. The parameters were chosen in such a way that the case in figure 4(a) has $\delta$ very different from $\delta^{*}$ and the case in figure $4(b)$ has $\delta$ close to $\delta^{*}$. These cases exemplify the different behaviours that are encountered during the edge-flame collapse. The case corresponding to figure $4(a)$ shows that there is a rapid initial transition from $\varphi_{o}(s)$ 


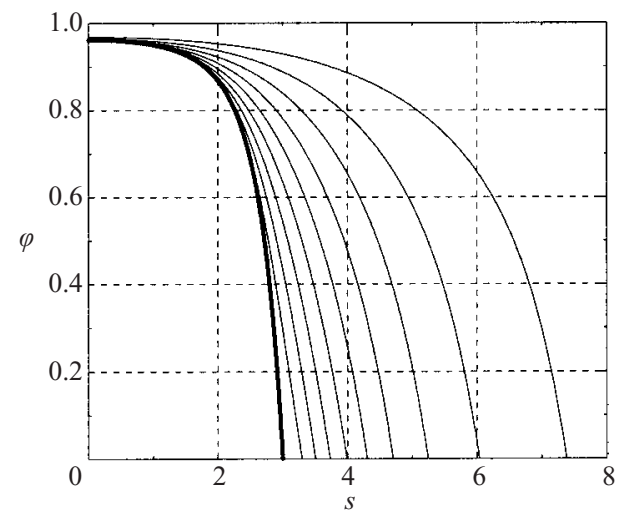

FIGURE 5. Evolution of $\varphi$ with time for axisymmetric case of an expanding edge-flame with $\delta=1, s_{f}(0)=3$ and $C=1$.

at $\tau=0$ towards a profile that, then, evolves more gradually at later times. This is caused by the large difference between $\delta$ and $\delta^{*}$, since the boundary condition of (3.6) requires that the derivative of $\varphi$ at $s=s_{f}$ must be equal to $-\delta$. The large difference between $\delta$ and $\delta^{*}$ creates a boundary layer at the interface to enforce the boundary condition and forces a rapid temporal transition. On the other hand, in figure $4(b)$ the profiles evolve smoothly with time because $\delta$ is close to $\delta^{*}$.

Inspection of figure 4 reveals a notable feature of the edge-flame collapse: following an initial transient, the $\varphi$ profiles evolve on a time scale that depends on the initial conditions, but in a manner that is consistent with a self-similar evolution. More precisely, the $\varphi$ profiles remain nested inside each other as time progresses and the peak $\varphi$ decreases with time at what visually seems to be the same rate as that exhibited by the edge-flame position, $s_{f}(\tau)$. This behaviour agrees with the analytical finding of $\S 3.1$. Figure 5 shows the evolution of the $\varphi$ profiles with time for an axisymmetric expanding case with $\delta=1$ and $s_{f}(0)=3$. Again, the thick line in this figure corresponds to the initial condition. Figure 5 shows that $\varphi$ of an expanding edge flame behaves approximately as a travelling wave. For example, the value of $\varphi$ at $s=0$ changes very little with time whereas the front translates approximately with $s_{f}$, certainly at $s=s_{f}$.

Finally, figure 6 shows a comparison of the numerical solution with the asymptotic self-similar expansion of the edge-flame position with corrections up to second order for the cases shown in figure 4. In general, the numerical results support the conjecture that the final stages of the edge-flame collapse are self-similar, although the extension of the self-similar region varies from case to case. It is shown that, in all cases, the leading-order solution, $s_{f_{0}}$, is a good approximation for the collapse of the edge-flame. The addition of the corrections, $a_{2}$ and $a_{3}$, usually gives better approximations, but the improvement achieved by incorporating higher-order corrections to the leading-order solution is, in general, marginal and it is case dependent. Figure 7 shows a comparison of $\varphi$ with the asymptotic solutions for the axisymmetric case with $C=1$, at a time when $s_{f}$ is equal to 0.1 . It is observed that the agreement between the asymptotic solution and the numerical solution is very good and it improves, in general, by increasing the number of terms of the expansion.

To date, we have focused on the results that correspond to the axisymmetric case. We address now the differences between the planar and axisymmetric configurations. It is 

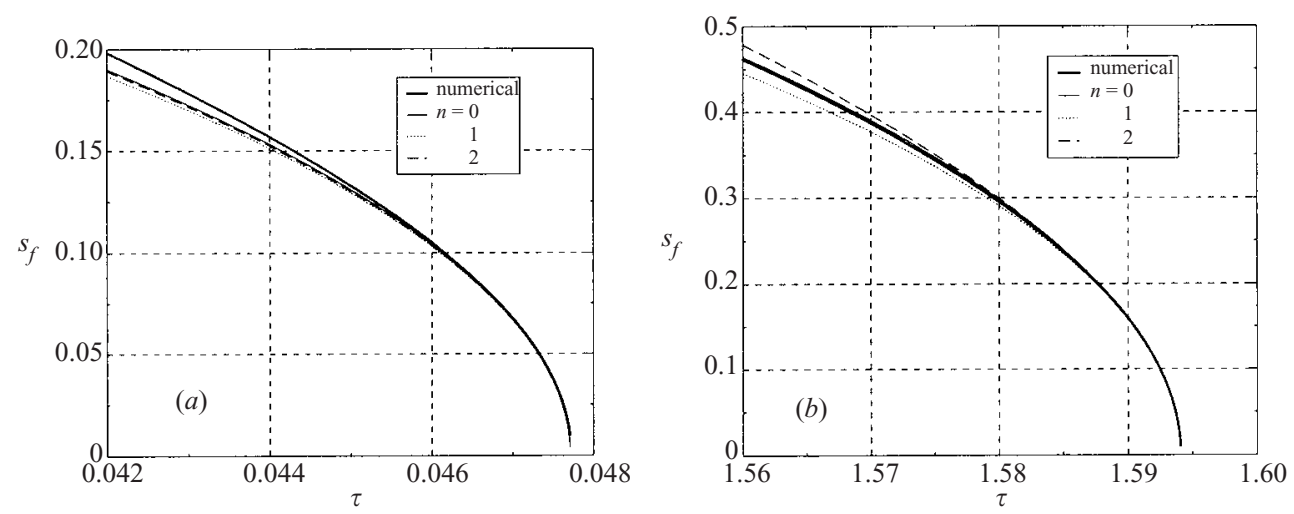

FIGURE 6. Evolution of the edge-flame position, $s_{f}$, with time obtained from numerical and asymptotic solutions for axisymmetric case with $C=1$ and $\delta=3,(a) s_{f}(0)=1$ and (b) $s_{f}(0)=3$. The index $n$ denotes the degree of the asymptotic approximation, $n=0$ is leading order, $n=1$ is leading order plus the first-order correction $\left(a_{2}\right)$ and $n=2$ is the leading order plus first- and second-order corrections $\left(a_{2}\right.$ and $\left.a_{3}\right)$.

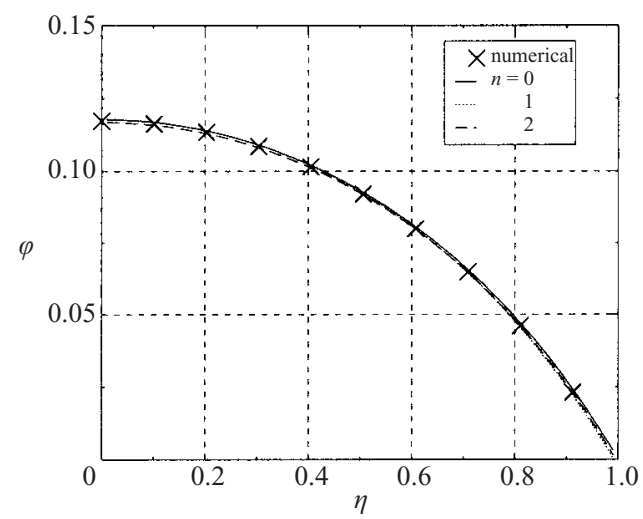

FIGURE 7. Profiles of $\varphi$ at a time when $s_{f}=0.1$ obtained from numerical and asymptotic solutions for axisymmetric case with $\delta=3, s_{f}(0)=3$ and $C_{3}=1$. The captions are identical to those of figure 6 .

found that there are no topological differences between the planar and axisymmetric cases. All collapsing planar cases showed self-similar behaviour and the same attracting and repulsing states discussed for the axisymmetric case. The numerical values are necessarily different, with some consequences. A general observation from table 1 is that the coefficients of the expansion of $s_{f}$ are smaller in the axisymmetric case than in the planar case, and the rate of collapse, proportional to $\sqrt{\Lambda}$, is $36 \%$ larger in the axisymmetric case. Another numerical difference between the planar and axisymmetric cases is that time to collapse, $\tau_{o}$, is generally larger in the planar case. The repulsive state that separates collapsing from expanding edge flames has higher values of $s_{f}$, for a given Damköhler number, in the axisymmetric case. In other words, the range of $s_{f}(0)$, for a fixed $\delta$, for which the flame hole collapses is larger in the axisymmetric case than in the equivalent planar case. 


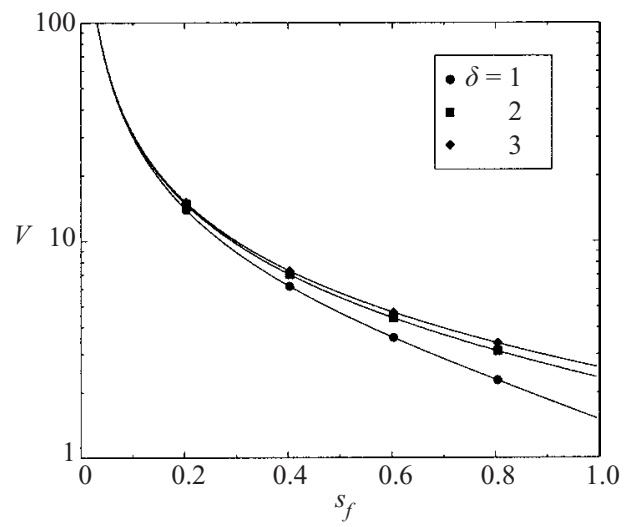

FiguRE 8. Dependence of the edge-flame velocity as a function of the edge-flame position for different reduced Damköhler numbers $(C=1)$.

\section{Discussion}

Our analysis of the structure of the edge-flame collapse can now be used to address some questions regarding the dependence of the solution on the Damköhler number, $\delta$. Buckmaster \& Jackson (2000) show that it is useful to plot the flame-edge velocity, $V=-\dot{s}_{f}$, against the edge position, removing the explicit time dependence. Taking the time derivative of (3.30) for $s_{f}$ and retaining the first two terms gives

$$
V=\Lambda\left(\frac{1}{s_{f}}+\frac{2 a_{2}}{\delta}\right),
$$

where $a_{2}$ is given in table 1 . Figure 8 shows a plot of $V$ against $s_{f}$ for three values of $\delta$. This result can be compared with figure 6 in Buckmaster \& Jackson (2000) that shows the same quantities obtained from the two-dimensional calculations of the problem from which the one-dimensional model is derived. The same qualitative behaviour is predicted by the analytic solution, that is, an increase of the edge-flame propagation velocity with increasing Damköhler number for constant edge-flame position. When $s_{f}$ is sufficiently small, the dependence on $\delta$ is subdominant to the singular collapse dynamics that are not dependent on the Damköhler number.

The present results describing the self-similar structure of the opposed edge-flame collapse were obtained for the particular case of unity Lewis number. In general, the Lewis number can differ from one in real flames. The self-similar solution is then not possible because no unique eigenvalue, $\Lambda$, can be defined in this case. Each field, temperature and mass fractions, defines a different eigenvalue through (3.23) when the Lewis number is not unity. When the Lewis number of, for example, the fuel, is close to one, with the Lewis number of the oxidizer equal to one, the self-similar solution may be appropriate in some circumstances. Unfortunately, the uniformly valid AEA with arbitrary order-unity Lewis number is not possible unless the convective-diffusive layer in front of the edge-flame and the diffusive-reactive layer at the edge are solved together iteratively.

Finally, we would like to comment on the fact that the present model is based on a constant density approximation. In real flames, density changes are substantial. The density takes typically a large value in the feeding streams and a low value at the flame. In the present problem, since the effects of flow divergence are not relevant to leading order, we expect that density effects will only affect the dominant balance of 
temporal and diffusive terms through the variation with temperature of the transport coefficients. In this case, the leading-order balance will be governed by

$$
\rho c_{p} \frac{\partial T}{\partial t}=\frac{1}{r} \frac{\partial}{\partial r}\left(\lambda r \frac{\partial T}{\partial r}\right),
$$

where $\lambda$ is typically approximated by a power-law function of the temperature and $c_{p}$ depends on both the composition of the mixture and the temperature. Since for sufficiently small flame holes radius $T \sim T_{a}$, the transport coefficients and density can be approximated by their hot (flame) values, we expect that to some extent the self-similar solution is relevant. On the other hand, the impact of variable density on the radius of validity of the self-similar solution discussed in this paper may be severely affected.

\section{Conclusion}

The non-stationary evolution of a one-dimensional model of opposed edge flames with algebraic side losses (Buckmaster 1996) embedded in a counterflow has been investigated. The importance of the counterflow lies in that it provides the conditions for a stationary mixing layer downstream of the flame hole $s>s_{f}$, where the diffusion flame resides. Two general cases are studied: a planar configuration and an axisymmetric configuration. Here, the evolution with time is studied for the case of unity Lewis number. The free-boundary problem is integrated numerically with a finite-difference technique and the evolution of the edge-flame position and mass fraction or temperature (equivalent in the unity Lewis number case) with time is discussed and compared with analytical findings.

Analysis of the final stages of the edge-flame collapse shows that it admits a self-similar structure. It is shown that, to leading order, the collapse is controlled by the balance of the temporal rate of change of the fuel mass fraction and diffusion. This results in an edge-flame position dependence that behaves as $\left(\tau_{o}-\tau\right)^{1 / 2}$, where $\tau_{o}$ is the time of collapse. The effect of side losses appear at the next order in an asymptotic expansion in terms of powers of $\left(\tau_{o}-\tau\right)^{1 / 2}$ and the effects of the counter flow are only relevant at the next order (second order). The fact that the side losses do not affect the leading-order solution makes possible inaccuracies in the model unimportant. Comparison between analytical and numerical results are favourable in all edge-flame collapses for both axisymmetric and planar flame holes. Both cases, planar and axisymmetric, are topologically identical and only the numerical values differ, owing to the presence of a curvature term in the diffusion term of the transport equation. For example, the self-similar analysis shows that the rate of collapse is approximately $36 \%$ larger in the axisymmetric case than in the planar case.

This work was supported by the Academic Strategic Alliances Program of the Accelerated Strategic Computing Initiative (ASCI/ASAP) under subcontract no. B341492 of DOE contract W-7405-ENG-48.

\section{Appendix. The stationary solutions}

The general problem of interest here consists of obtaining solutions of the equation

$$
s \frac{\mathrm{d} \varphi}{\mathrm{d} s}-\left(\frac{\mathrm{d}^{2} \varphi}{\mathrm{d} s^{2}}+\frac{j}{s} \frac{\mathrm{d} \varphi}{\mathrm{d} s}\right)-C(1-\varphi)=0 .
$$


An appropriate change of variables, $\xi=\frac{1}{2} s^{2}$, reduces (A 1) to Kummer's equation (Abramowitz \& Stegun 1972), from which the general solution of (A 1) can be obtained as

$$
\varphi=1-c_{0} M\left(\frac{1}{2} C, b, \frac{1}{2} s^{2}\right)-c_{1} U\left(\frac{1}{2} C, b, \frac{1}{2} s^{2}\right),
$$

where $M(a, b, z)$ and $U(a, b, z)$ are the confluent hypergeometric functions. The parameter $b$ is equal to

$$
b=\frac{1}{2}(j+1) \text {, }
$$

and takes the value $\frac{1}{2}$ for the planar case and 1 for the axisymmetric case.

Solutions of this form have been obtained previously by Nayagam et al. (1999) and Nayagam \& Williams (2001) in axisymmetric edge flames propagating at small velocities. It can be seen that these solutions involving the confluent hypergeometric functions apply to a more general family of problems, that is, planar and axisymmetric edge flames in counterflow. Also note that these solutions resemble those describing stationary cylindrical premixed flames, Echekki \& Ferziger (1993), and also involve confluent hypergeometric functions. Important properties of Kummer's functions $M$ and $U$ are

$$
\begin{gathered}
\frac{\mathrm{d} M}{\mathrm{~d} z}(a, b, z)=\frac{a}{b} M(a+1, b+1, z), \\
\frac{\mathrm{d} U}{\mathrm{~d} z}(a, b, z)=-a U(a+1, b+1, z), \\
M(a, b, z \rightarrow 0)=1, \\
U(a, b, z \rightarrow 0)= \begin{cases}\frac{\Gamma(1-b)}{\Gamma(1+a-b)} & 0<b<1, \\
-\frac{\ln z+\psi(a)}{\Gamma(a)} & b=1 .\end{cases}
\end{gathered}
$$

The only relevant outer solution of $\varphi$ is the left solution. In this case, $\varphi^{\prime}(0)=0$ and $\varphi\left(s_{f}\right)=0$. The solution to (A 1 ) is then given by (A 2) with $c_{1}=0$ because $U$ is singular at $s=0$. We then obtain

$$
\varphi\left(C, s_{f}, s\right)=1-\frac{M\left(\frac{1}{2} C, b, \frac{1}{2} s^{2}\right)}{M\left(\frac{1}{2} C, b, \frac{1}{2} s_{f}^{2}\right)},
$$

where the boundary condition at $s=s_{f}$ has been used to determine the value of the constant $c_{0}$. From this expression, the value of $\varphi$ at the origin, $s=0$, is equal to

$$
\varphi\left(C, s_{f}, 0\right)=1-\frac{1}{M\left(\frac{1}{2} C, b, \frac{1}{2} s_{f}^{2}\right)},
$$

and the derivative of (A 7) with respect to $s$, is

$$
\varphi^{\prime}\left(C, s_{f}, s\right)=-\frac{C s}{2 b} \frac{M\left(1+\frac{1}{2} C, b+1, \frac{1}{2} s^{2}\right)}{M\left(\frac{1}{2} C, b, \frac{1}{2} s_{f}^{2}\right)} .
$$

\section{REFERENCES}

Abramowitz, M. \& Stegun, I. (Eds.) 1972 Handbook of Mathematical Functions, 10th edn. Dover. Buckmaster, J. 1996 Edge-flames and their stability. Combust. Sci. Tech. 115, 41-68. 
Buckmaster, J. \& Jackson, T. 2000 Holes in flames, flame isolas, and flame edges. Proc. Combust. Inst. 28, 1957-1964.

Buckmaster, J. \& Short, M. 1999 Cellular instabilities, sublimit structures and edge-flames in premixed counterflows. Combust. Theory Modelling 3, 199-214.

Daou, J. \& LiÑ́́n, A. 1998 The role of unequal diffusivities in ignition and extinction fronts in strained mixing layers. Combust. Theory Modelling 2, 449-477.

ECHeKki, T. \& Chen, J. 1996 The effects of complex chemistry on triple flames. Center for Turbulence Research, Proc. Summer Program, Stanford, CA, 1996, pp. 217-234.

ECHEKKI, T. \& Ferziger, J. 1993 Studies of curvature effects on laminar premixed flames: stationary cylindrical flames. Combust. Sci. Tech. 90, 231-252.

Ghosal, S. \& Vervisch, L. 2000 Theoretical and numerical study of a symmetrical triple flame using the parabolic flame path approximation. J. Fluid Mech. 415, 227-260.

Kioni, P., RogG, B., BraY, K. \& Liñán, A. 1993 Flame spread in laminar mixing layers: the triple flame. Combust. Flame 95, 276.

Ko, Y. \& Chung, S. 1999 Propagation of unsteady tribrachial flames in laminar non-premixed jets. Combust. Flame 118, 151-163.

LIÑÁN, A. 1974 The asymptotic structure of counterflow diffusion flames for large activation energies. Acta Astronaut. 1, 1007-1039.

Nayagam, V., Balasubramaniam, R. \& Ronney, P. 1999 Diffusion flame-holes. Combust. Theory Modelling 3, 727-742.

Nayagam, V. \& Williams, F. 2001 Diffusion-flame dynamics in von Kármán boundary layers. Combust. Flame 125, 974-981.

Nayagam, V. \& Williams, F. 2002 Lewis-number effects on edge-flame propagation. J. Fluid Mech. 458, 219-228.

Ruetsch, G., Vervisch, L. \& Liñán, A. 1995 Effects of heat release on triple flames. Phys. Fluids 7, 1447-1454.

SAntoro, V., Liñán, A. \& GomeZ, A. 2000 Propagation of edge flames in counterflow mixing layers: experiments and theory. Proc. Combust. Inst. 28, 2039-2046.

ShaY, M. \& Ronney, P. 1998 Nonpremixed edge flames in spatially varying straining flows. Combust. Flame 112, 171-180.

Short, M., Buckmaster, J. \& Kochevets, S. 2001 Edge-flames and sublimit hydrogen combustion. Combust. Flame 125, 893-905.

Thatcher, R. \& Dold, J. 2000 Edges of flames that do not exist: flame-edge dynamics in a non-premixed counterflow. Combust. Theory Modelling 4, 435-457.

Vedarajan, T. \& Buckmaster, J. 1998 Edge-flames in homogeneous mixtures. Combust. Flame 114, $267-273$.

Williams, F. A. 1985 Combustion Theory. Benjamin/Cummings. 